performed. Many appointments may therefore be suitable for community services or by telemedicine, thus freeing up capacity and responsiveness of in-person specialist clinics for patients with more complex needs, worsening symptoms or new diagnoses.

Conflict of Interest Dr Singhal's salary is funded by a fellowship from Abbott

\section{IS A DEDICATED HFPEF CLINIC EFFECTIVE IN MANAGING PATIENTS WITH HEART FAILURE WITH PRESERVED FRACTION (HFPEF)?}

Patrick Tran, Thomas Long, Jessica Smith, Prithwish Banerjee. University Hospitals Coventry and Warwickshire, Coventry, UK

\subsection{6/heartjnl-2021-BCS.134}

Background HFpEF is often diagnosed in elderly patients coupled with comorbidities and degrees of frailty. Given the lack of evidence-based therapies, the common maxim is to 'optimize comorbidities with a holistic approach,' Joint cardiology-geriatric HFpEF clinics have been recommended but are unlikely to be sustainable with current resource constraints. To improve outcome a dedicated HFpEF clinic led by HF specialists was therefore piloted to include specific interventions: diuretics, fluid restriction $(<1.5 \mathrm{~L} /$ day $)$, heart rate control $(<70 /$ min for sinus and $<80 / \mathrm{min}$ for atrial fibrillation (AF)), blood pressure (BP) control $(<130 / 80 \mathrm{mmHg})$, cardioversion for $\mathrm{AF}$, exercise recommendation and control of non-cardiovascular comorbidities (e.g. diabetes, COPD, obesity).

Methods A single-centre retrospective analysis of $202 \mathrm{HFpEF}$ out-patients was performed over a 12-month period. Diagnosis of HFpEF was based on a local diagnostic algorithm similar to the HFA-PEFF score recommended by ESC. Electronic and paper records were reviewed, capturing comorbidities, clinic interventions and follow-up. We also performed a pilot analyses of hospitalization and mortality rates between patients managed in the general HF clinic and in the dedicated HFpEF clinic.

Results The study population reflected a typical HFpEF profile (mean age $79 \pm 9.6$ years, 55\% female, NYHA Class III/IV (48\%), hypertension (78\%), AF, 53\%, chronic kidney disease (41\%), ischaemic heart disease (30\%) and obesity (13\%) (table 1). Clinic time was limited to 15-20 minutes. The majority of clinic interventions were cardiology-focused, namely congestion management (64\%), drug reviews (86\%) of anti-hypertensives, and rate/rhythm control of AF (48\%). With the current outpatient structure, there was little remaining time to consider non-cardiac comorbidities. Although not powered in the pilot analysis, preliminary data showed no difference in hospitalization and mortality rates between general $\mathrm{HF}$ and dedicated HFpEF clinics. In both clinics, $25 \%$ of patients had at least two admissions at 12 months mainly due to non-cardiac causes (table 2).

Conclusion Structure of specialized HFpEF clinics needs to be reshaped to meet the complex demands of HFpEF and improve patient outcomes. Current clinic activities concentrated on managing fluid balance, $\mathrm{BP}$ and cardiac drug reviews with little emphasis on comorbidities e.g. optimizing diabetes, anaemia or addressing polypharmacy which are common causes for re-hospitalization. We believe that our outpatient clinic reflects most HF clinics in the UK. Accordingly, an efficient HFpEF clinic pathway needs to be formulated. Quality improvement ideas include (1) screening patients before clinic for any significant comorbidities to enable early specialist advice or referral (2) entrusting HF nurse specialists to

Abstract 137 Table 1 Baseline characteristics of patients

\begin{tabular}{|c|c|c|c|}
\hline Demographics & $\begin{array}{l}\text { HFpEF clinic } \\
(\mathrm{N}=101)\end{array}$ & $\begin{array}{l}\text { General clinic } \\
\qquad(N=101)\end{array}$ & P Value \\
\hline Age- year (mean $\pm \mathrm{SD})$ & $78 \pm 9.8$ & $79 \pm 9.4$ & 0.38 \\
\hline Female sex- no (\%) & $57(56.4)$ & $55(54.5)$ & 0.89 \\
\hline Average number of clinic visits (IQR) & $1(1-2)$ & $1(1-2)$ & 0.07 \\
\hline \multicolumn{4}{|l|}{ Number of clinic visits } \\
\hline 1 & 68 & 56 & \\
\hline 2 & 24 & 29 & \\
\hline 3 & 6 & 14 & \\
\hline 4 & 3 & 1 & \\
\hline 5 & 0 & 1 & \\
\hline \multicolumn{4}{|l|}{ Co-morbidities- no (\%) } \\
\hline Hypertension & $78(77.2)$ & $79(78.2)$ & 0.87 \\
\hline Coronary artery disease & $28(27.7)$ & $33(32.7)$ & 0.44 \\
\hline Atrial fibrillation & $51(50.5)$ & $56(55.4)$ & 0.48 \\
\hline Type II Diabetes (T2DM) & $31(30.7)$ & $36(35.6)$ & 0.46 \\
\hline Valvular heart disease & $25(24.8)$ & $20(19.8)$ & 0.40 \\
\hline Chronic kidney disease (estimated GFR<60) & $38(37.6)$ & $44(43.6)$ & 0.39 \\
\hline Chronic obstructive pulmonary disease & $11(10.9)$ & $11(10.9)$ & 1.00 \\
\hline Obesity (BMI $\geq 30$ ) & $17(16.8)$ & $10(9.9)$ & 0.15 \\
\hline \multicolumn{4}{|l|}{ NYHA functional class- no (\%) } \\
\hline I & 5 & 6 & 1.00 \\
\hline II & 53 & 42 & 0.17 \\
\hline III & 39 & 53 & 0.07 \\
\hline IV & 4 & 0 & 0.12 \\
\hline
\end{tabular}


Abstract 137 Table 2 Subgroup analysis of hospital admission - $n(\%)$ at 6 and 12 months

\begin{tabular}{|c|c|c|c|}
\hline & $\begin{array}{l}\text { HFpEF clinic } \\
(\mathrm{N}=101)\end{array}$ & $\begin{array}{l}\text { General HF clinic } \\
\qquad(N=101)\end{array}$ & P Value \\
\hline Total number of hospital admissions at 6 months & 65 & 53 & 0.36 \\
\hline Cardiovascular admissions- no (\%) & $21(32.3)$ & $21(39.6)$ & 1.00 \\
\hline Non-cardiovascular admissions- no (\%) & $44(67.7)$ & $32(60.4)$ & 0.22 \\
\hline Total number of hospital admissions at 12 months & 103 & 112 & 0.15 \\
\hline Cardiovascular admissions- no (\%) & $34(33.7)$ & $45(40.2)$ & 0.74 \\
\hline Non-cardiovascular admissions- no (\%) & $69(68.3)$ & $67(59.8)$ & 0.84 \\
\hline Patients with $\geq 2$ hospitalization over 12 months & 26 & 26 & 1.00 \\
\hline Decompensated HF & 11 & 15 & \\
\hline Community-acquired pneumonia & 5 & 4 & \\
\hline Postural hypotension related falls & 2 & 4 & \\
\hline Symptomatic Anaemia & 3 & 2 & \\
\hline Acute kidney injury & 5 & 1 & \\
\hline
\end{tabular}

oversee current clinic activities allowing cardiologists more time for a holistic approach (3) adapting virtual clinics for regular follow-ups.

Conflict of Interest None

\section{APPLICABILITY OF USE OF SODIUM- GLUCOSE CO- TRANSPORTER-2 INHIBITORS (SGLT2I) IN PATIENTS WITH HEART FAILURE WITH REDUCED EJECTION FRACTION (HFREF) AT LONDON NORTHWEST UNIVERSITY HEATHCARE NHS TRUST}

Oltiana Lakra, Angelica Sharma, Zahraa Al-Dulaimi, Jaskaran Sethi, Elaine Hui, Jaymin S Shah. London North West University Healthcare NHS Trust, London, UK

\subsection{6/heartjnl-2021-BCS.135}

Background Heart failure (HF) on maximal therapy has a high morbidity and mortality associated with it. An estimated 26 million individuals are affected by HF worldwide resulting in a high cost expenditure. SGLT2i have prognostic benefit in patients with HFrEF on top of conventional therapies, irrespective of glycaemic status. Recently the NICE guidelines have recommended Dapagliflozin (SGLT2I) as an option for treating patients with symptomatic $\mathrm{HFrEF}$ as an add-on to optimised standard care. We aimed to estimate the proportion of patients, presenting with HF, that would be eligible for SGLT2i within our busy district general hospital and moreover to create and disseminate local guidelines for initiating SGLT2i treatment.

Methods We conducted a retrospective review of patients presenting at our hospital with HF between April - August 2020 $(n=251)$. Patients over 18 , with or without type 2 diabetes (T2D), EF $\leq 40 \%$ and $e G F R \geq 30$ were included as eligible for SGLT2i. Patients with type 1 diabetes (T1D) and death during admission were excluded. 101/251 (40\%) individuals had HFrEF. Parameters evaluated were demographics, eGFR, HbA1C, NT-ProBNP alongside regular medications.

Results 89\% ( $\mathrm{n}=90 / 101)$ met the inclusion criteria (68 $(67 \%)$ male, mean $( \pm$ SD) age $71.3( \pm 13.4) .11(10 \%)$ had eGFR $<30$ and $1(1 \%)$ had T1D, hence were excluded. $36(37 \%)$ had T2D. The median (IQR) HbA1c was 52 (40-56) mmol/ mol, mean $( \pm$ SD) eGFR $51( \pm 20) \mathrm{mL} / \mathrm{min} / 1.73 \mathrm{~m} 2$ and median (IQR) NT-ProBNP $7482 \quad(3117.5-18512.8) \quad \mathrm{pg} / \mathrm{mL}$. Median (IQR) length of hospitalization was 6 (3.5 - 11) days.
Mortality was $10.9 \%(n=11 / 101)$ at 3-months. On discharge, HF therapy included Angiotensin-converting enzyme inhibitor/ Angiotensin receptor blocker/Angiotensin receptor-neprilysin inhibitor (78\%), Beta-blocker (89\%), Mineralocorticoid-receptor antagonist (40\%) and diuretic (91\%)). Based on previous studies, SGLT2i would reduce mortality by $2.3 \% \quad(n=2$ patients in this cohort).

Conclusion Our study reveals that up to 9 in 10 individuals with HFrEF are suitable for SGLT2i. Poor renal function is a limiting factor. To achieve optimal success, intensive education for patients and multi-disciplinary team on benefits and possible complications with a designed pathway is essential. Conflict of Interest none

\section{WHAT HAPPENS TO PATIENTS CODED HEART FAILURE AND DISCHARGED FROM THE EMERGENCY DEPARTMENT}

Jan Oliver, Tom Harris, Sam McClure. Sunderland Royal Hospital, Sunderland, UK

\subsection{6/heartjnl-2021-BCS.136}

Heart failure is a common presentation to the emergency department, accounting for 5\% of all medical admissions. Evidence from the national audit data base and our own local data confirms reduced mortality with specialist heart failure management. In our institution we recognized a group of patients that were seen in the emergency department and discharge without an admission and given a diagnosis of heart failure, these patients were not identified to our heart failure service. It is unclear if these patients have a formal diagnosis and what follow up is given. It is proposed that patients attending the emergency department and are considered fit for immediate discharge have sub optimal care. This study aims to evaluate the quality of care given to patients discharged from the emergency department where the primary diagnosis is heart failure by assessing three key performance indicators, NTproBNP, echocardiogram and follow up care.

Methods Attendances at the emergency department that were coded as having heart failure as a primary diagnosis during a six month period $(01.10 .18$ - 31.03.19) were reviewed. The coded diagnosis included heart failure, left ventricular failure, acute pulmonary oedema or cardiomyopathy. This 